\title{
Screening for Non-Alcoholic Fatty Liver Disease (NAFLD) among Obese and Overweight Children: Prevalence and predictors
}

\section{Srinivasan Thiagarajan}

IGMC and RI: Indira Gandhi Medical College and Research Institute

\section{Sadhanandham Shrinuvasan}

IGMC and RI: Indira Gandhi Medical College and Research Institute

Thirunavukkarasu Arun Babu ( $\square$ babuarun@yahoo.com )

All India Institute of Medical Sciences (AllMS) https://orcid.org/0000-0003-1123-5270

\section{Research Article}

Keywords: Non Alcoholic Fatty Liver Disease (NAFLD), Obesity, Overweight, Children, Liver steatosis, Transaminasemia, metabolic risk factors, insulin resistance, HOMA-IR, Fatty liver

Posted Date: June 2nd, 2021

DOl: https://doi.org/10.21203/rs.3.rs-555820/v1

License: (9) This work is licensed under a Creative Commons Attribution 4.0 International License. Read Full License

Version of Record: A version of this preprint was published at Indian Journal of Gastroenterology on January 21st, 2022. See the published version at https://doi.org/10.1007/s12664-021-01198-0. 


\section{Abstract}

Background: Non- alcoholic fatty liver disease (NAFLD) is increasingly recognised in obese and overweight children. NAFLD is the most common cause of chronic liver disease in these children. Objectives: To assess the prevalence of NAFLD and to identify the anthropometric, metabolic risk factors for NAFLD.

Materials and methods: This cross sectional study was done with 154 overweight and obese children. The study population was divided into 2 groups based on the presence or absence of NAFLD by screening with ultrasonography and/or alanine transaminase levels. Anthropometric (Body mass index, waist circumference) and biochemical parameters (Total cholesterol, triglyceride, high density lipoprotein, low density lipoprotein, very low density lipoprotein, Insulin, Homeostasis model assessment of insulin resistance (HOMA-IR), alanine transaminase, aspartate transaminase) were compared between these two groups.

Results: NAFLD was diagnosed based on abnormal screening in $79(51.3 \%)$ overweight and obese children.. No age or gender difference was noted between 2 groups. NAFLD patients did not differ significantly from patients without NAFLD in age, Body mass index, systolic blood pressure, diastolic blood pressure and waist circumference. LDL Cholesterol levels were found significantly higher in the NAFLD group. There was no significant difference found in lipid profile apart from LDL cholesterol, aspartate transaminase and HOMA-IR between 2 groups.

Conclusion: NAFLD is common in overweight and obese South Indian children. High LDL cholesterol level is a risk factor for NAFLD in these children. Screening of this high risk group for early diagnosis of NAFLD is essential to prevent and monitor further progression of the disease.

\section{Introduction}

The incidence of obesity among children has seen a steep rise in the recent years in developing countries like India [1]. Childhood obesity is associated with co-morbidities like asthma, sleep apnea, fatty liver disease, gallstones, gastro-esophageal reflux and non-alcoholic fatty liver disease (NAFLD) [2]. Moreover, children with obesity are more likely to become obese adults and are at higher risk of developing early onset non-communicable diseases during adulthood like type 2 diabetes, hypertension, polycystic ovarian disease, coronary vascular disease etc $[3,4]$. NAFLD is the commonest liver disease encountered by obese children and adolescents [5]. NAFLD is characterized by increased fat deposits in liver along with inflammation and liver cell damage [6]. Though early stages of NAFLD are reversible, progressive involvement can lead to steatohepatitis, fibrosis and cirrhosis [7]. However, only minority of obese children progress to severe stages beyond steatosis. According to multiple hit hypothesis theory, several factors cause progression of NAFLD in addition to genetic predisposition like insulin resistance, hormones secreted by adipose tissue, gut microbiome, nutrition etc. [8]. Detecting NAFLD early is 
extremely crucial in preventing the progression to further stages of disease by dietary and life style interventions in obese children.

NAFLD in children is associated with chronic systemic disorders like hypertension, dyslipidaemia, increased risk of metabolic syndrome, type 2 diabetes, and cardiovascular diseases [9].NAFLD is also feature of several inborn errors of metabolism like Wilsons's disease, Galactosemia, glycogen storage disorders, hereditary fructose intolerance, a1-antitrypsin deficiency, lipid storage disorders like NiemannPick disease, fatty acid oxidation disorders etc. [10]. Many research studies over the last decade have linked NAFLD with the development of insulin resistance and metabolic syndrome in obese children [11, 12]. Though many modalities like ultrasonogram (US), computerised tomography (CT), magnetic resonance imaging (MRI) are used commonly to diagnose NAFLD, liver biopsy is the gold standard test to diagnose NAFLD in children [13]. MRI has been considered as the most sensitive non-invasive method of detecting steatosis in early stages [14]. Nevertheless, due to practical limitations, screening for NAFLD is commonly done by US or alanine transaminase (ALT) levels in clinical settings $[15,16]$. For diagnosing NAFLD, using two times the gender-specific ALT (ALT $\geq 44$ for girls and $\geq 50$ for boys) in obese and overweight children aged $\geq 10$ years has a sensitivity and specificity of $88 \%$ and $26 \%$ respectively [ 17 , 18].

The prevalence of NAFLD in South Indian overweight and obese children is unknown due to asymptomatic evolution of steatosis and non-availability of published data. Hence this study was done to assess the prevalence of NAFLD among south Indian obese children and to identify the predictive anthropometric and metabolic risk factors for NAFLD.

\section{Methodology}

This cross sectional study was done out between January 2019 to December 2019 in a Tertiary care teaching Hospital in South India. Institutional ethics committee clearance was obtained before enrolment. Informed consent from parents/guardian and assent from older children were sought before inclusion. All children attending outpatient department aged 5 to 13 years with body mass index (BMI) equivalent to adults more than $23 \mathrm{~kg} / \mathrm{m} 2$ were included. Children with adult equivalent BMI of more than $27 \mathrm{Kg} / \mathrm{m}^{2}$ and between $23-27 \mathrm{~kg} / \mathrm{m}^{2}$ were classified as obese and overweight respectively [19]. Children with acute febrile illness, chronic liver disease, history of receiving hepatotoxic drugs were excluded.

Anthropometric parameters like weight, height, waist circumference were measured. Standardized Electronic weighing machine (EQUAL Digital, Front and back and red LED double display, $0.1-120 \mathrm{~kg}, \mathrm{MS}$ platform, unique power technologies) was used for weight measurement. Height was measured using stadiometer. BMI was calculated using Quetelet index as the ratio of weight in $\mathrm{kg}$ and square of height in meters. Waist circumference (WC) was measured in upright posture at the midpoint between lower margin of the last rib and upper border of iliac crest using a non-stretchable tape to the nearest $0.1 \mathrm{~cm}$. Systolic and diastolic blood pressure (SBP, DBP) was measured in the right arm by korotkoff method using sphygmomanometer after 5 minutes of rest in the sitting position. US of liver was done using 
Philips HD 7 xe with curvilinear probe 2-5 Hz. Liver steatosis was graded as mild, moderate, severe based on the echogenicity compared to right renal cortex [20]. From an overnight fasting blood sample, glucose, total cholesterol (TC), triglycerides (TG), high density lipoprotein (HDL) cholesterol, Aspartate transaminase (AST) and ALT were estimated using standard reagent kits. From the triglyceride value, low density lipoprotein (LDL) and very low density lipoprotein (VLDL) cholesterol was calculated using friedwald's formula. Fasting insulin was estimated by chemiluminescence method. Homeostasis model assessment of insulin resistance (HOMA-IR) [21] was measured by using the formula HOMA-IR = fasting glucose $(\mathrm{mg} / \mathrm{dl}) \times$ fasting insulin $(\mathrm{ulU} / \mathrm{ml}) / 405$. NAFLD was diagnosed by liver US and/or elevated ALT levels $\geq 50 \mathrm{U} / \mathrm{l}$ (boys) $\geq 44 \mathrm{U} / \mathrm{l}$ (girls). Though various studies used different cut off levels for ALT [22], widely accepted threshold level for ALT is 2 times the upper limit of gender-specific ALT (ALT $\geq 50$ for boys and $\geq 44$ for girls) was used in this study [17]. Hepatitis B surface antigen, IgM antibody hepatitis $A$ virus, serum ceruloplasmin levels and ophthalmological examination to rule out Kayser Fleisher ring were done for all children with transaminasemia (ALT levels $>35 \mathrm{IU} / \mathrm{L}$ ). ALT was repeated after 3 months only for those with ALT $\geq 35 \mathrm{IU} / \mathrm{L}$.

The sample size was calculated using $\mathrm{G}^{\star}$ Power 3: A flexible statistical power analysis program for the social, behavioral and biomedical sciences [23]. The required sample size was 146 using Mann-whitney $\mathrm{U}$ test for the comparison of mean between two groups with an effect size of 0.5 , with 0.90 beta error and 0.05 alpha error.

Included cases were divided into 2 groups based on screening for NAFLD done by US and ALT levels (> twice the upper limit). All anthropometric, biochemical parameters were compared between the 2 study groups. The statistical software (SPSS 24) was used for data analysis. Descriptive data were expressed as mean, median and standard deviations (SD) for continuous variables. Mann-Whitney $U$, and t test were used to compare continuous variables. Chi-square test was applied for categorical variables. The receiver operating characteristics (ROC) analysis was used to verify the independent variables and to define an appropriate cut-off. $p$ value less than 0.05 was considered as statistically significant.

\section{Results}

A total of 154 overweight and obese children were recruited in the study. It included 73 boys and 81 girls between 5 to $13 \mathrm{yrs}$. Mean age of boys and girls were $9.72( \pm 1.99)$ and $10.06( \pm 2.08)$, respectively. The anthropometric and biochemical characteristics of the study population stratified by gender are summarized in Table - 1. No family history of liver disease was found in any overweight/obese child. $112(72.72 \%)$ children had history of obesity/overweight in either or both parents. US features of fatty liver were diagnosed in 73 patients ( 38 girls and 35 boys) out of which 63 and 10 had mild and moderate steatosis respectively. Out of 10 children with moderate steatosis, 9 were obese. Elevated ALT was found in 13 patients ( 8 boys and 5 girls). When either US finding and the criteria of ALT > 50(boys), > 44 (girls) were considered, it was found that $79(51.3 \%)$ had NAFLD. Seven children had both US steatosis and transaminasemia. Four children had ALT levels more than $80 \mathrm{IU} / \mathrm{L}$. Among 37 obese children and 117 overweight children, NAFLD was found in 19 and 60 children respectively. No statistical significant 
difference ( $p=0.994)$ was found in NAFLD diagnosis between overweight and obese children. No difference between baseline and repeat ALT levels was found in children with transaminasemia $(\geq 35$ IU/L). Though mean ALT levels were higher in the obese when compared with overweight children (34.12 vs 32.92), it was statistically insignificant $(p=0.072)$. LDL cholesterol levels were found to be significantly higher in the NAFLD group $(p=0.033)$. The anthropometric and biochemical characteristics of the NAFLD and non-NAFLD group are summarized in Table -2 .

The correlation analysis between $\mathrm{BMI}$ and other study parameters among patients with NAFLD showed positive relationship found between $\mathrm{BMI}$ and WC $r=0.598, p<0.001, \mathrm{BMI}$ and Insulin $r=0.214, p<0.05$, $\mathrm{BMI}$ and HOMO-IR $r=0.225, p<0.05$. No relationship was found between BMI and Cholesterol, Triglycerides, AST, ALT, Insulin, HDL, LDL and VLDL $(p>0.05)$. The correlation analysis between BMI and other study parameters among patients without NAFLD showed positive association between $\mathrm{BMI}$ and WC $(r=0.487, p<0.001), \mathrm{BMI}$ and AST $(r=0.334, p<0.05)$. There was no relationship found between BMI and Cholesterol, Triglycerides, ALT, Insulin, HOMO-IR, HDL, LDL and VLDL ( $p>0.05)$.

Comparison of ROC curves for parameters between NAFLD and non-NAFLD showed that we could not establish a cut off of each variable for identifying children at high risk of NAFLD since very low area under curve was obtained for all the studied parameters.

\section{Discussion}

Dyslipidemia and insulin resistance are a consequence of abnormal lipid metabolism and hepatic lipid accumulation in obese individuals. The mechanism of hepatocellular damage and development of NAFLD though unclear, is commonly attributed to the lipotoxicity of high circulating levels of free fatty acid, total cholesterol and other lipid metabolites. These lipid products in turn contribute to mitochondrial dysfunction due to oxidative stress and free radical damage. The current management strategy for NAFLD in children focuses on weight reduction and normalising lipid levels [24, 25].

Many studies have used different modalities to diagnose NAFLD in children as there is no consensus regarding the diagnostic criteria. US technique sensitivity ranges from $60-94 \%$ and a specificity of $84-$ $100 \%$ in detecting steatosis [18].Sensitivity and specificity of US increases to $100 \%$ and $90 \%$ respectively when hepatic steatosis content exceeds $20 \%$ [26].In the present study, NAFLD diagnosis was based on liver steatosis in US and/or more than 2 times the upper limit of gender specific ALT [17]. The $61.7 \%$ prevalence of NAFLD among obese and overweight children in our study is similar to other published studies from India using similar diagnostic modalities [27, 28].

No significant difference in BMI, SBP, DBP and WC between NAFLD and non-NAFLD group were noted. This observation is discordant with findings of many western and Indian studies [27-29]. Pawar et al found that systolic hypertension was the sole independent factor associated with NAFLD [27]. Another study found that BMI, WC, SBP, DBP were significantly higher in NAFLD group among 11-16 year old children [28]. 
Though we observed apparent increase of non HDL lipid levels (TC, TG, LDL, VLDL) in NAFLD group, significant statistical difference was found only with LDL. Though some studies demonstrated increased lipid levels like cholesterol, triglycerides in NAFLD patients, the findings are not consistent [27-32]. On similar lines, HDL cholesterol was observed to be lesser in the NAFLD group compared to the non-NAFLD group without statistically significant difference. Pawar et al demonstrated higher triglyceride and AST levels in NAFLD children [27]. Two similar studies also found significant association between low HDL levels and NAFLD $[29,32]$. Though many studies have confirmed the association of insulin resistance with NAFLD $[8,28,29]$, Pawar et al [27] found no difference in insulin and HOMA-IR levels between NAFLD and non-NAFLD group which is similar to our study. This finding can be partly explained by the relative younger pre-pubertal age group of the study population compared to adolescent age group in many other studies as the progression of insulin resistance is directly proportional to the increasing age in children. Our study population included children less than 13 years of age whereas many studies included children till 16 to 18 years of age.

Since most patients were asymptomatic except for Obesity and overweight, liver biopsy was not done to evaluate NAFLD. MRI is considered as better imaging modality to confirm NAFLD in children. MRI proton density fat fraction (MRI-PDFF) is superior in classifying grades of steatosis based on a recent metaanalysis in 2019 with pooled sensitivity and specificity of MRI-PDFF for grades 0 vs. $1-3,0-1$ vs. 2-3, and $0-2$ vs. 3 were 0.93 and $0.94,0.74$ and 0.90 , and 0.74 and 0.87 , respectively [14]. Due to practical limitations in a resource limited setup and poor patient affordability, MRI imaging could not be performed to diagnose NAFLD. Other limitations of this study include lack of non-obese controls and not using fibroscan, a specialized ultrasound to assess steatosis and fibrosis of NAFLD. Since more sensitive methods for diagnosis of NAFLD were not used in this study, the results obtained are different and discordant from the published data in literature.

\section{Conclusion}

NAFLD is common in overweight and obese South Indian children based on screening by US and ALT. High LDL cholesterol level is a risk factor for NAFLD in overweight and obese children. Screening of this high risk group for early diagnosis of NAFLD is essential to prevent and monitor further progression of the disease.

\section{Highlights}

\section{What is already known?}

- NAFLD is common obese and overweight children

- Risk factors and prevalence of NAFLD in Indian obese and overweight children not fully known 
- NAFLD is common (61.7\%) in overweight and obese South Indian

- High LDL cholesterol level is a risk factor for NAFLD in overweight and obese children.

\section{What are the future clinical and research implications of the study findings?}

- Screening of high risk group for early diagnosis of NAFLD is essential

- Early identification of NAFLD can help monitoring and limiting further progression

\section{References}

1. Bhave S, Bavdekar A, Otiv M, IAP National Task Force for Childhood Prevention of Adult Diseases. Childhood Obesity. IAP National Task Force for Childhood Prevention of Adult Diseases: Childhood Obesity. Indian Pediatr. 2004;41:559-75.

2. Halfon N, Kandyce L, Slusser W. Associations between obesity and comorbid mental health, developmental, and physical health conditions in a nationally representative sample of US children aged 10 to 17. Academic Pediatrics. 2013;13:6-13.

3. Lloyd LJ, Langley-Evans SC, McMullen S. Childhood obesity and risk of the adult metabolic syndrome: a systematic review. Int J Obes (Lond). 2012;36:1-11.

4. Thiagarajan S, ArunBabu T, Manivel P. AcanthosisNigricans and Metabolic Risk Factors in Obese Children. Indian J Pediatr. 2020;87:162.

5. Giorgio V, Prono F, Graziano F, Nobili V. Pediatricnon alcoholic fatty liver disease: old and new concepts on development, progression, metabolic insight and potential treatment targets. BMC Pediatr. 2013;13:40.

6. Anderson EL, Howe LD, Jones HE, Higgins JP, Lawlor DA, Fraser A. The Prevalence of Non-Alcoholic Fatty Liver Disease in Children and Adolescents: A Systematic Review and Meta-Analysis. PLoS One. 2015;10:e0140908.

7. Prokopowicz Z, Malecka-Tendera E, Matusik P. Predictive Value of Adiposity Level, Metabolic Syndrome, and Insulin Resistance for the Risk of Nonalcoholic Fatty Liver Disease Diagnosis in Obese Children. Can J GastroenterolHepatol. 2018;9465784.

8. Buzzetti E, Pinzani M, Tsochatzis EA. The multiple-hit pathogenesis of non-alcoholic fatty liver disease (NAFLD). Metabolism. 2016;65:1038-48.

9. Gökçe S, Atbinici Z, Aycan Z, Çinar HG, Zorlu P. The relationship between pediatricnon alcoholic fatty liver disease and cardiovascular risk factors and increased risk of atherosclerosis in obese children. Pediatric Cardiology. 2013;34:308-15.

10. Yıldız Y, Sivri HS. Inborn errors of metabolism in the differential diagnosis of fatty liver disease. Turk J Gastroenterol. 2020;31:3-16.

11. Lonardo A, Ballestri S, Marchesini G, Angulo P, Loria P. Nonalcoholic fatty liver disease: a precursor of the metabolic syndrome. Digestive Liver Disease. 2015;47:181-90. 
12. Kasturiratne A, Weerasinghe $S$, Dassanayake AS. Influence of non-alcoholic fatty liver disease on the development of diabetes mellitus. Journal of Gastroenterology Hepatology. 2013;28:142-7.

13. Yoneda M, Imajo K, Takahashi H, Ogawa Y, Eguchi Y, Sumida Y, et al. Clinical strategy of diagnosing and following patients with nonalcoholic fatty liver disease based on invasive and noninvasive methods. J Gastroenterol. 2018;53:181-96.

14. Gu J, Liu S, Du S, Zhang Q, Xiao J, Dong Q, et al. Diagnostic value of MRI-PDFF for hepatic steatosis in patients with non-alcoholic fatty liver disease: a meta-analysis. EurRadiol. 2019;29:3564-73.

15. Draijer LG, Feddouli S, Bohte AE, et al. Comparison of diagnostic accuracy of screening tests ALT and ultrasound for pediatric non-alcoholic fatty liver disease. Eur J Pediatr. 2019;178:863-70.

16. Koplay M, Sivri M, Erdogan H, Nayman A. Importance of imaging and recent developments in diagnosis of nonalcoholic fatty liver disease. World J Hepatol. 2015;7:769-76.

17. Vos MB, Abrams SH, Barlow SE, Caprio S, Daniels SR, Kohli R, et al. NASPGHAN Clinical Practice Guideline for the Diagnosis and Treatment of Nonalcoholic Fatty Liver Disease in Children: Recommendations from the Expert Committee on NAFLD (ECON) and the North American Society of Pediatric Gastroenterology, Hepatology and Nutrition (NASPGHAN). J PediatrGastroenterolNutr. 2017;64:319-34.

18. Schwimmer JB, Newton KP, Awai HI, Choi LJ, Garcia MA, Ellis LL, et al. Paediatric gastroenterology evaluation of overweight and obese children referred from primary care for suspected non-alcoholic fatty liver disease. Aliment PharmacolTher. 2013;38:1267-77.

19. Khadilkar V, Yadav S, Agrawal KK, Tamboli S, Banerjee M, Cherian A, et al. Revised IAP growth charts for height, weight and body mass index for 5- to 18-year-old Indian children. Indian Pediatr. 2015;52:47-55.

20. Shannon A, Alkhouri N, Carter-Kent C, Monti L, Devito R, Lopez R, et al. Ultrasonographic quantitative estimation of hepatic steatosis in children with NAFLD. J PediatrGastroenterolNutr. 2011;53:190-5.

21. Kurtoğlu S, Hatipoğlu N, Mazıcıoğlu M, Kendirici M, Keskin M, Kondolot M. Insulin resistance in obese children and adolescents: HOMA-IR cut-off levels in the prepubertal and pubertal periods. J Clin Res PediatrEndocrinol. 2010;2:100-6.

22. Vajro P, Lenta S, Socha P, Dhawan A, McKiernan P, Baumann U,et al. Diagnosis of non alcoholic fatty liver disease in children and adolescents: position paper of the ESPGHAN Hepatology Committee. J PediatrGastroenterolNutr. 2012;54:700-13.

23. Faul F, Erdfelder E, Lang AG, Buchner A. G*Power 3: a flexible statistical power analysis program for the social, behavioral, and biomedical sciences. Behav Res Methods. 2007;39:175-91.

24. Perla FM, Prelati M, Lavorato M, Visicchio D, Anania C. The Role of Lipid and Lipoprotein Metabolism in Non-Alcoholic Fatty Liver Disease. Children (Basel). 2017;4:46.

25. Clemente MG, Mandato C, Poeta M, Vajro P. Pediatric non-alcoholic fatty liver disease: Recent solutions, unresolved issues, and future research directions. World J Gastroenterol. 2016;22:807893. 
26. Dasarathy S, Dasarathy J, Khiyami A. Validity of real time ultrasound in the diagnosis of hepatic steatosis: a prospective study. J Hepatol. 2009;51:1061-7.

27. Pawar SV, Zanwar VG, Choksey AS, Mohite AR, Jain SS, Surude RG, et al. Most overweight and obese Indian children have nonalcoholic fatty liver disease. Ann Hepatol. 2016;15:853-61.

28. Jain $V$, Jana $M$, Upadhyay B, Ahmad N, Jain O, Upadhyay AD, et al. Prevalence, clinical \& biochemical correlates of non-alcoholic fatty liver disease in overweight adolescents. Indian J Med Res. 2018;148:291-301.

29. Yang HR, Chang EJ. Insulin resistance, body composition, and fat distribution in obese children with nonalcoholic fatty liver disease. Asia Pac J ClinNutr. 2016;25:126-33.

30. Deeb A, Attia S, Mahmoud S, Elhaj G, Elfatih A. Dyslipidemia and Fatty Liver Disease in Overweight and Obese Children. J Obes. 2018; 8626818.

31. Alkhouri N, Eng K, Lopez R, Nobili V. Non-high-density lipoprotein cholesterol (non-HDL-C) levels in children with nonalcoholic fatty liver disease (NAFLD). Springer plus. 2014;3:407.

32. Alkassabany YM, Farghaly AG, El-Ghitany EM. Prevalence, risk factors, and predictors of nonalcoholic fatty liver disease among schoolchildren: a hospital-based study in Alexandria, Egypt. Arab J Gastroenterol. 2014;15:76-81.

\section{Tables}

Table 1: Anthropometric characteristics and Biochemical parameters of Study Population 


\begin{tabular}{|c|c|c|c|c|c|c|c|}
\hline \multirow[t]{2}{*}{ Variable } & \multicolumn{2}{|c|}{$\begin{array}{l}\text { All } \\
(n=154)\end{array}$} & \multicolumn{2}{|l|}{$\begin{array}{l}\text { Male } \\
(n=73)\end{array}$} & \multicolumn{2}{|l|}{$\begin{array}{l}\text { Female } \\
(n=81)\end{array}$} & \multirow[t]{2}{*}{$\begin{array}{l}\mathrm{t} \text { test } \\
\mathrm{p} \text { value }\end{array}$} \\
\hline & Mean & SD & Mean & SD & Mean & SD & \\
\hline BMI $\left(\mathrm{kg} / \mathrm{m}^{2}\right)$ & 23.64 & 3.02 & 24.22 & 3.19 & 23.11 & 2.78 & $0.022 *$ \\
\hline $\mathrm{SBP}(\mathrm{mmHg})$ & 107.46 & 9.61 & 107.97 & 8.79 & 107 & 10.32 & 0.532 \\
\hline $\mathrm{DBP}(\mathrm{mmHg})$ & 71.38 & 7.29 & 72.21 & 6.59 & 70.64 & 7.83 & 0.181 \\
\hline WC $(\mathrm{cm})$ & 75.51 & 7.72 & 76.80 & 8.08 & 74.34 & 7.23 & 0.048* \\
\hline Cholesterol (mg/dl) & 154.90 & 30.96 & 154.17 & 33.29 & 155.55 & 28.89 & 0.784 \\
\hline $\mathrm{TG}$ (mg/dl) & 107.83 & 54.84 & 100.90 & 51.37 & 114.07 & 57.38 & 0.137 \\
\hline $\mathrm{HDL}(\mathrm{mg} / \mathrm{dl})$ & 34.85 & 7.08 & 34.76 & 7.43 & 34.76 & 7.43 & 0.890 \\
\hline LDL(mg/dl) & 98.19 & 23.78 & 97.35 & 25.12 & 98.94 & 22.64 & 0.681 \\
\hline VLDL(mg/dl) & 19.57 & 8.87 & 18.75 & 8.01 & 20.31 & 9.57 & 0.278 \\
\hline AST (IU/L) & 38.20 & 15.75 & 40.01 & 18.96 & 36.56 & 12.05 & 0.176 \\
\hline ALT (IU/L) & 34.06 & 15.84 & 35.68 & 18.38 & 32.60 & 13.10 & 0.230 \\
\hline $\begin{array}{l}\text { Insulin } \\
(\mu \mathrm{IU} / \mathrm{ml})\end{array}$ & 10.80 & 5.23 & 9.81 & 5.00 & 11.70 & 5.30 & $0.025^{*}$ \\
\hline HOMA - IR & 1.68 & 1.12 & 1.51 & 1.00 & 1.84 & 1.21 & 0.071 \\
\hline
\end{tabular}

BMI- Body mass index; SBP-Systolic blood pressure; DBP- Diastolic blood pressure; WC- Waist circumference; TG- Triglyceride; HDL- High density lipoprotein; LDL- Low density lipoprotein; VLDL- Very low density lipoprotein; AST- Aspartate transaminase; ALT- Alanine transaminase; HOMA-IR- Homeostasis model assessment of insulin resistance. 
Table 2: Comparison of Anthropometry and Biochemical parameters of obese and overweight children with and without NAFLD.

\begin{tabular}{|c|c|c|c|c|c|c|c|c|}
\hline \multirow[t]{2}{*}{ Variables } & \multicolumn{3}{|c|}{$\begin{array}{l}\text { NAFLD } \\
(n=79)\end{array}$} & \multicolumn{3}{|c|}{ Non-NAFLD group } & \multirow[t]{2}{*}{ Mann-Whitney U } & \multirow[t]{2}{*}{$p$ value } \\
\hline & Mean & SD & Median & Mean & SD & Median & & \\
\hline Age (years) & 10.13 & 2.03 & 10.20 & 9.65 & 2.03 & 10.00 & 2628.00 & 0.226 \\
\hline $\mathrm{BMI}\left(\mathrm{kg} / \mathrm{m}^{2}\right)$ & 24.17 & 3.02 & 24.43 & 23.08 & 2.96 & 22.50 & 2382.50 & $0.036^{*}$ \\
\hline SBP (mmHg) & 108.80 & 9.34 & 110.00 & 106.05 & 9.75 & 108.00 & 2442.50 & 0.052 \\
\hline DBP (mmHg) & 71.87 & 7.11 & 70.00 & 70.88 & 7.49 & 70.00 & 2624.50 & 0.194 \\
\hline $\mathrm{WC}(\mathrm{cm})$ & 76.72 & 8.05 & 76.00 & 74.24 & 7.19 & 74.00 & 2469.00 & 0.074 \\
\hline Cholesterol (mg/dl) & 160.24 & 36.46 & 156.00 & 149.28 & 22.77 & 150.00 & 2450.00 & 0.064 \\
\hline $\mathrm{TG}(\mathrm{mg} / \mathrm{dl})$ & 113.14 & 62.60 & 101.00 & 102.24 & 45.01 & 95.00 & 2704.50 & 0.351 \\
\hline $\mathrm{HDL}(\mathrm{mg} / \mathrm{dl})$ & 34.23 & 7.82 & 33.00 & 35.51 & 6.20 & 36.00 & 2465.00 & 0.071 \\
\hline $\mathrm{LDL}(\mathrm{mg} / \mathrm{dl})$ & 102.67 & 26.55 & 102.00 & 93.47 & 19.56 & 94.00 & 2372.50 & $0.033^{*}$ \\
\hline VLDL(mg/dl) & 20.16 & 9.11 & 19.00 & 18.95 & 8.64 & 17.00 & 2681.00 & 0.308 \\
\hline AST (IU/L) & 39.15 & 19.01 & 35.00 & 37.20 & 11.41 & 36.00 & 2877.00 & 0.757 \\
\hline Insulin $(\mu \mathrm{IU} / \mathrm{ml})$ & 10.70 & 5.31 & 10.00 & 10.91 & 5.18 & 10.00 & 2901.50 & 0.825 \\
\hline HOMA - IR & 1.80 & 1.17 & 1.60 & 1.57 & 1.08 & 1.20 & 2588.50 & 0.176 \\
\hline
\end{tabular}

BMI- Body mass index; SBP-Systolic blood pressure; DBP- Diastolic blood pressure; WC- Waist circumference; TG- Triglyceride; HDL- High density lipoprotein; LDL- Low density lipoprotein; VLDL- Very low density lipoprotein; AST- Aspartate transaminase; ALT- Alanine transaminase; HOMA-IR- Homeostasis model assessment of insulin resistance. 


\section{Declarations}

\section{Author Contributions:}

ST and TA conceived the study. ST, SS and TA collected data and managed the patients. ST reviewed the literature and drafted the initial version of the manuscript. All authors contributed to drafting of the manuscript and approved the final version of the manuscript. TA shall act as guarantor of the paper.

Funding: None

Conflict of interest: Nil

Ethical clearance : No 06/IEC/IGMC\&RI/F-7/2018

\section{Acknowledgement:}

We would like to thank Mr. Durairaj Mahadevan, Dept of Biochemistry for his assistance in statistical analysis 